\title{
NASA Structure and Evolution of the Universe Theme: Science Overview
}

\author{
Nicholas White \\ Laboratory for High Energy Astrophysics \\ NASA's Goddard Space Flight Center \\ Greenbelt, MD 20771 \\ 301-286-8801 \\ nwhite(a)lheapop.gsfc.nasa.gov \\ and \\ Bruce Margon \\ Astronomy Department, University of Washington \\ Seattle, Washington \\ margon@atlas.astro.washington.edu
}

\begin{abstract}
The NASA Office of Space Science Structure and Evolution of the Universe (SEU) theme covers a wide variety of scientific investigations, from the nearest bodies to the farthest observable distances just after the time of the Big Bang. SEU supports experiments that sense radiation of all wavelengths, together with particle and gravitational wave detection. Recently completed road mapping and strategic planning exercises have identified a number of near- and medium-term space initiatives for the 2003-2023 time frame. Each of these experiments pushes the state of the art technically, but will return incredible new insights on the formation and evolution of the universe, as well as probe fundamental laws of physics in regimes never before tested. The scientific goals and technological highlights of each mission are described.
\end{abstract}

\section{TABLE OF CONTENTS}

1. INTRODUCTION

2. TOP-PRIORITY SEU MISSIONS

3. MIDTERM SEU MISSIONS

4. CONCLUSIONS

\section{INTRODUCTION}

The Structure and Evolution of the Universe (SEU) theme at NASA's Office of Space Science (OSS) addresses the largest and smallest scales of the universe, from the largest physical entities known to subatomic particles. The missions identified as a part of this program seek to explore the darkest and brightest places yet to be identified, from black holes to gamma ray bursts, and senses every type of radiation, from radio to gamma rays, as well as cosmic rays and gravitational waves.

Two of the three NASA "Great Observatories" launched thus far have been SEU programs - Compton Gamma Ray Observatory (CGRO) and Chandra X-ray Observatory
(CXO, formerly AXAF); and a major gamma ray mission, GLAST, is in an advanced stage of development.

The Far Infrared and Submillimeter Telescope (FIRST), a European Space Agency (ESA) cornerstone mission with substantial NASA participation, is also moving forward. A vigorous program of smaller Explorer class missions has and will continue to be a continuing foundation of the SEU program.

Recently the Structure and Evolution of the Universe Subcommittee (SEUS) of the OSS Space Science Advisory Committee (SScAC) completed a plan for major missions covering the next 25 years of SEU activities (Cosmic Journeys: To the Edge of Gravity. Space and TimeStructure and Evolution of the Universe Roadmap. 20032023, NP-1999-11-184-GSFC, 1999). These SEU "roadmap" missions have in turn been incorporated into a NASA OSS "Enterprise Strategic Plan," due for publication in December 2000

In the 2003-2007 time frame, SEUS has identified three top-priority near-term science objectives, together with missions to accomplish these goals. The three problems span a diverse range of complimentary subdisciplines, observational technique, timescales, and cost, thus forming a coherent core program for SEU in this interval.

In the later 2008-2013 period six further missions have come to the top of the roadmapping process. We refer to these as the "Midterm Missions." These missions address high priority science, but their selection and prioritization pose special challenges. Their precise progress towards launch will depend on the rate of development of cuttingedge technologies, which may be difficult to predict accurately, and whose scientific goals may evolve as new, relevant results arrive over the next decade. In many cases these new technologies are applicable elsewhere in the 
NASA program, as well as in areas totally unrelated to space science.

\section{TOP-PRIORITY SEU MISSIONS}

The three highest priority SEU missions are Constellation$X$, Laser Interferometer Space Antenna (LISA), and Advanced Cosmic Ray Composition Experiment for the Space Station (ACCESS).

Constellation- $X$ will obtain precise measures of the chemical composition and physical conditions of objects ranging from the closest stars to the most distant quasars via $\mathrm{x}$-ray spectroscopy of unprecedented sensitivity.

LISA will utilize, for the first time, gravitational radiation to study supermassive black holes throughout the Universe, compact binary sources within our Galaxy, and a possible gravitational wave background. LISA will consist of a 5 million $\mathrm{km}$ arm-length laser interferometer in space.

ACCESS will determine the nature of the highest energy cosmic rays, one of the most important questions in this fundamental field by measuring the characteristics of individual elements over a wide range of mass and energy, utilizing the International Space Station (ISS) as a platform.

Each of these missions will challenge the state of art in a variety of technologies, techniques, and/or management and integration issues. Precision $x$-ray optics, picometer metrology, and detectors of unprecedented spectral resolution, quantum efficiency, and noise rejection are required. Although each mission has been described in detail in other venues, including this meeting, a few scientific and technical highlights of each are presented here.

\section{Constellation- $X$}

Optical astronomy transitioned into astrophysics more than a half century ago when it became routinely possible to obtain spectra with resolution of $\lambda / \Delta \lambda \approx 3000$. Velocities of hundreds of kilometers per second, ubiquitous in both galactic and extragalactic objects in the universe, then became measurable, and key line multiplets of common nuclei could be resolved to yield quantitative plasma diagnostics. As x-ray astronomy approaches its half-century anniversary imaging capabilities have far outrun spectroscopy, and $\mathrm{x}$-ray astronomers by at least this criterion have not yet entered the routine spectroscopic era. There are indeed a handful of x-ray spectra of this high resolution currently available, and in the next decade we can expect Chandra and XMM-Newton to raise this inventory to at least several dozen objects of each astrophysical class. This will be a fabulous harvest, teaching us how to use $\mathrm{x}$ ray spectra for quantitative analysis, just as the optical astronomer learned decades earlier. But just as the optical astronomer would hardly be satisfied being confined spectroscopically to naked eye stars, x-ray astronomy can hardly flourish when it is confined to the very brightest few dozen examples of each class, leaving us unable to judge neither the physical diversity of objects nor their evolution with cosmic time.

Constellation-X will provide a hundred-fold increase in sensitivity over the high-resolution spectroscopy capabilities of current missions such as Chandra and XMM-Newton. Constellation- $X$ is the $x$-ray astronomy equivalent of large ground-based optical telescopes such as the Keck and the VLT, complementing the high spatial resolution capabilities of Chandra. The targets of Constellation-X are not speculative. They will be largely well cataloged by the current generation of sensitive imaging missions

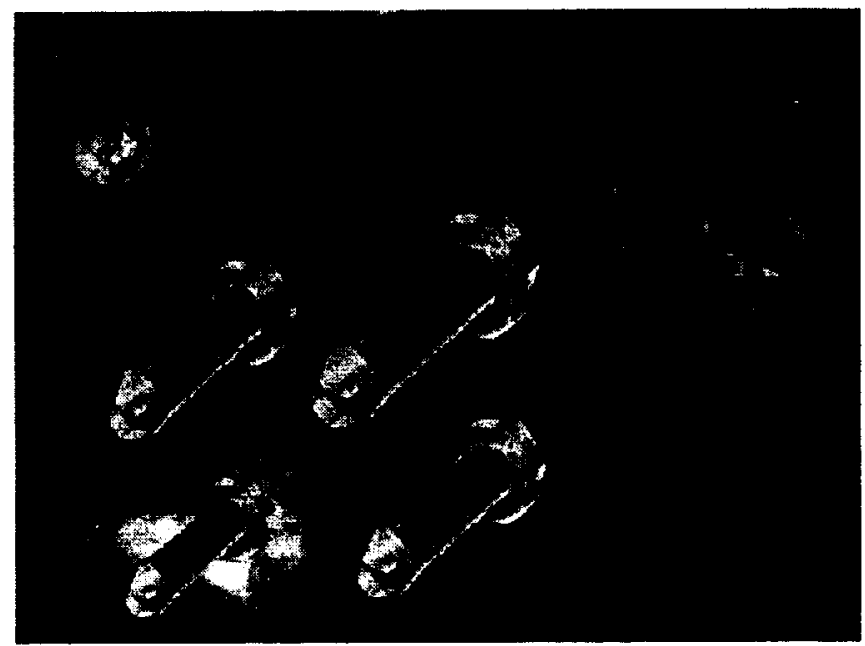

Figure 1. An artists impression of the four Constellation-X satellites at the Sun-Earth L2 point (approximately 1.5 million $\mathrm{km}$ from the Earth, in the anti-sun direction. By utilizing a multi-satellite design to achieve large collecting area, the overall mission risk can be reduced and a production line approach employed to reduce cost.

Constellation- $X$ will address questions concerning the extremes of gravity and the evolution of the universe. X-ray observations of broadened iron emission lines in Active Galactic Nuclei will measure black hole mass and rotation, exploiting general relativistic effects occurring in the strong gravity limit. Constellation-X will trace black hole evolution with cosmic time and provide new insight into the contribution of accretion processes to the total energy output of the universe. By looking across a broad range of red shifts, Constellation- $\mathrm{X}$ will reveal the formation epoch of clusters of galaxies and relate the observations to current models of galaxy formation. Current inventories indicate that many baryons predicted by Big Bang nucleosynthesis and subsequent stellar processing seem to be missing, and Constellation- $\mathrm{X}$ will search for them- for example. in a hot 
metal-enriched intergalactic medium. Constellation-X will identify large numbers of $x$-ray spectral lines in stellar coronae, supernova remnants, and the interstellar medium, providing essential information on chemical enrichment processes, as well as detailed measures of plasma temperature, pressure, and density over a wide range of astrophysical settings.

Constellation-X was included in the 1997 OSS Strategic Plan as a candidate New Start for 2004, requiring significant technology investment in a number of areas. Notwithstanding limited funding to date, very substantial progress has been made in several key areas, including light-weight $\mathrm{x}$-ray mirrors, improved energy resolution of $\mathrm{x}$ ray microcalorimeters, multilayer depositions for hard $x$-ray telescopes, CZT detectors for hard $\mathrm{x}$-rays, and the overall multisatellite approach to the mission. With additional technology investments to sustain this rapid progress over the next few years, Constellation- $\mathrm{X}$ will be ready for a new start in 2005.

\section{Laser Interferometer Space Antenna}

One of the last channels to be opened is that of gravitational radiation. Gravitational waves are propagating polarized gravitational fields that can be detected by measuring the change they cause in the distance between separated test masses. Gravitational waves are generated at detectable levels only by massive astrophysical sources undergoing violent dynamics. Once generated, the waves propagate unaffected by scattering or absorption due to intervening matter, making them difficult to detect. It also means that they give information on strong-gravity and high-velocity astrophysical regions not obtainable by other means. Although waveforms have not yet been directly detected, there is clear evidence that gravitational radiation exists. The observed orbital decay of the radio pulsar PSR $1913+16$ is precisely consistent with the predicted energy loss due to gravitational radiation.

There are several ground-based projects for detecting gravitational waves, including the U.S. Laser Interferometer Gravitational Wave Observatory (LIGO), which will be sensitive to waves with $\approx 10 \mathrm{~Hz}$ to $\mathrm{kHz}$ frequencies. Such waves are produced by such objects as supernovae, coalescing neutron stars, and coalescing stellar-mass black holes. Another very interesting spectral range is the millihertz region (signal periods of minutes to hours). Here the strongest prototypical source might be two coalescing massive black holes in an AGN. Because of seismic and gravity gradient noise backgrounds it is impossible to measure these low-frequency waves from the ground.

LISA is a space-borne gravitational wave observatory that will be sensitive to these lower frequency waves (Figure 2). It will consist of three spacecraft orbiting the sun in an equilateral triangle formation with $5 \times 10^{6} \mathrm{~km}$ sides. The center of the triangle is in the ecliptic, $1 \mathrm{AU}$ from the sun, trailing about $20^{\circ}$ behind the Earth. Using laser interferometry in the relatively quiet space environment, LISA will detect low-frequency gravitational waves by monitoring tiny distance changes between proof masses within each spacecraft. LISA will be sensitive in the 0.1 $\mathrm{mHz}$ to $1 \mathrm{~Hz}$ frequency range (i.e., much lower in frequency than LIGO). LISA will thus complement ground-based gravitaional-wave observatories; the huge difference in frequencies means that there is no overlap between the expected sources in the low (LISA) and high (LIGO) frequency bands.

The LISA scientific objectives are:

- to observe the gravitational radiation signature from merging supermassive black holes in galactic nuclei

- to observe gravitational radiation from compact stars scattered into capture orbits around supermassive black holes

- to take a census of all short-period compact galactic binaries in our galaxy

- to search for a gravitational wave background from the early universe and to search for unexpected sources of gravitational waves

For periodic sources LISA will achieve angular resolution of $\approx 0.5^{\circ}$ (strong sources determined from the amplitude and phase modulation of the signal as the LISA array orbits the sun) and strain sensitivity (with one-year integration times) of $\approx 10^{-23}$ at frequencies of a few milliHz.



Figure 2. The three LISA spacecraft will contain proof masses and provide a drag free environment. They will detect gravitational waves by precise ranging between the proof masses to 10 pico-meters, every second. 
Three key technologies are needed to make LISA a reality. First, inertial sensors, with proof masses isolated from nongravitational forces, must be employed. Second, microthrusters are required to keep each spacecraft centered on its proof mass. Third, laser metrology is needed to measure sub-picometer changes in distance between widely separated proof masses.

The LISA concept is the product of a large international collaboration. LISA will be a cooperative project of NASA and ESA. The LISA concept was strongly endorsed in the 1990 NASA Report of the Ad Hoc Committee on Gravitation Physics, in the joint NAS/NRC (1988) report on Space Science in the Twenty First Century: Imperatives of the Decades 1995-2015, and in ESA reports concerning their future program.

\section{Advanced Cosmic-Ray Composition Experiment for the Space Station}

Cosmic rays are an important part of the dynamics and structure of our galaxy. Their impact is largely hidden from us, because their magnetic fields and shock structures with which they interact are not directly visible to us, so we must study them by taking detailed measurements on the arriving particles themselves. The population of cosmic rays contains all the known long-lived charged particles and has an average energy density similar to that of the magnetic fields and photons in our galaxy. The overall distribution of cosmic ray energies is remarkable in that it is almost a constant power law over at least 13 decades in energy and 31 decades in flux.

The current view of the origin of most of these cosmic rays is stochastic acceleration of particles at the shock fronts produced by supernovae. Although recent $x$-ray and high energy gamma-ray observations have shown nonthermal concentrations of electrons near such shocks, the strongest argument for these ideas on the origin of the bulk of cosmic rays remains simple energetics: only supernovae can supply the enormous power needed to sustain the population of cosmic rays.

ACCESS is an instrument designed to explore directly the connection of cosmic rays with supernovae. A small steepening, or "knee," in the slope of the power law energy spectrum of cosmic rays near $10^{15} \mathrm{eV}$ is thought to be associated with the maximum possible energies achievable by direct supernova shock acceleration of cosmic rays. ACCESS has a large enough collecting power to measure directly the particles near these energies, previously detected only by the air showers they produced in the Earth's atmosphere. The direct measurements can provide the crucial missing information on how the fluxes of each type of cosmic ray nucleus vary at high energy; this is not possible with air showers. This approach gives an essential added dimension of enormous value, similar to that added by spectroscopy in visible light measurements.

The individual elemental spectra can be used to determine directly the source spectra of the cosmic ray nuclei. The simplest theory of shock acceleration expects all these spectra to be similar power-law forms that seem inconsistent with current measurements. ACCESS can study these spectra with unprecedented accuracy over more than four decades in energy using a single instrument. There are known to be features associated with atomic energy scales in the elemental composition of the cosmic-ray sources relative to galactic material. ACCESS can determine if these features persist to the highest energies. ACCESS can directly measure the cosmic-ray energy spectra into the knee region. A direct study can be made of the characteristic signatures of the upper limits of supernova shock acceleration

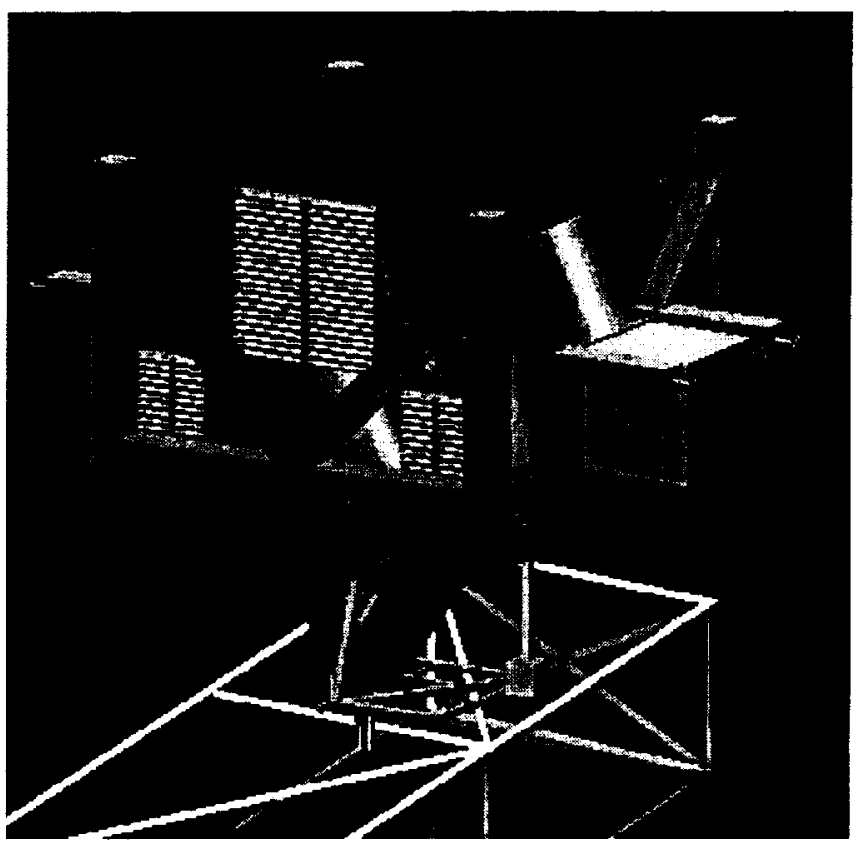

Figure 2. An artists impression of the ACCESS instrument attached to the ISS.

These individual fluxes can also be used to reconstruct the history of cosmic rays in our Galaxy. We already know that at lower energies cosmic rays escape more easily from the plane of the Galaxy as their energy increases. We also know this kind of behavior cannot continue to the high energies of the knee region since large anisotropies in arrival direction would be produced which are not observed. ACCESS will have detectors sensitive enough to discover how this transition occurs.

To collect enough of the very high-energy cosmic rays ACCESS must be a very large instrument, with several square meters of effective collecting area. To provide an 
energy estimate for lighter cosmic rays ACCESS must also contain a calorimeter. These features combine to make ACCESS by necessity a large and heavy instrument for spaceflight. The lack of accurate pointing requirements make this instrument a good match to the experimental facilities of the ISS, where the Space Shuttle can easily lift massive payloads to the attachment points. ACCESS is designed for a zenith-pointed site on ISS, consisting of the combination of a large area transition radiation detector with a hadronic calorimeter (Figure 3 ). It is designed to measure individual elements over the range of $1 \leq Z \leq 28$ and up to energies of $\approx 10^{15} \mathrm{eV}$.

\section{SEU MIDTERM MISSIONS}

SEUS selected six Midterm Missions (launch in 20082013) for its roadmap. A first subgroup of three has our highest priority recommendation in each of three subdisciplines: $x$-ray, gamma-ray, and far IR astronomy. These missions will be the "seed corn" of each of these fields: ambitious goals tackling the most basic and important science, destined to keep each field vigorous and moving forward through the second decade of the millennium. These missions, noted in order of increasing wavelength, are the High-resolution Spectroscopic Imager (HSI) mission for gamma-ray astronomy, a MicroArcsecond X-ray Imaging Mission (MAXIM) technology pathfinder, and a bold program in large farinfrared optics, applicable to both 10 -m class filled aperture arrays as well as $30-\mathrm{m}$ baseline interferometers.

The second group of Midterm Missions includes three further programs, each of which tackles essential scientific problems. The Orbiting Wide Angle Light Collectors (OWL) will provide dramatic new capabilities in cosmic ray astronomy, the Energetic X-ray Imaging Survey Telescope (EXIST) will complete the first all-sky map of the celestial sphere in hard $\mathrm{x}$-rays, and the Advanced Radio Interferometer between Space and Earth (ARISE) mission will probe the radio sky with unprecedented angular resolution.

\section{CONCLuSIONS}

Astronomy and Astrophysics Survey Committee Recommendations

Although the SEUS obtained wide community input while developing its current roadmap, including a half-dozen working groups charged with intensively canvassing their colleagues in specific fields, clearly no one document can claim to contain the infallible wisdom of the discipline. In astronomy and astrophysics, a much broader survey of scientific priorities and approaches to challenge them is made approximately once per decade under the auspices of the National Research Council. The resulting reports have been popularly referred to as the "Decadal Surveys," and they have proven to be of crucial importance in demonstrating to federal funding agencies that the community has cogently undergone the painful prioritization process. Over the past three to four decades, a gratifyingly large fraction of the programs recommended in the Decadal Surveys has come to fruition.

An advance copy of the latest Decadal Survey, entitled Astronomy and Astrophysics in the New Millennium, was released to the public in May 2000 and is scheduled for final publication at the end of 2000. As with previous editions, this one will probably be referred to by the names of its lead authors, i.e., the McKee-Taylor Report. SEUS was gratified to see that this independent and prestigious report agrees with the conclusions of the SEUS roadmap, both broadly and in detail. The three high priority missions discussed here, Constellation-X, LISA, and ACCESS, place well in the very highest priority missions featured in McKee-Taylor. Specifically, in large spaceborne initiatives, Constellation- $X$ is second in priority only to Next Generation Space Telescope (NGST), and in moderate initiatives, LISA is second only to GLAST, another SEU program that is well underway. Smaller programs were not prioritized by the McKee-Taylor report, but ACCESS is described prominently and repeatedly in that document. All six of the six SEU Midterm Missions are also featured and discussed in this latest Decadal Survey.

\section{Acknowledgements}

The authors thank the SEUS scientists who formulated the SEU science roadmap: J. Armstrong (JPL), A. Davidsen (JHU), C. Forman (CfA), G. Garmire (PSU), N. Gehrels (NASA/GSFC), J. Hewitt (MIT), M. Kamionkowski (Caltech), C. Lawrence (JPL), D. Lester (UT), D. McCammon (Wisconsin), P. Michelson (Stanford), B. Peterson (OSU), S. Swordy (Chicago), H. Tananbaum (CfA), and A. Bunner (NASA Headquarters, SEUS Executive Secretary). The Roadmap Coordinator, without whom the effort simply would not have happened, was F. Harrison (Caltech). Invaluable support was provided by D. Kniffen (NASA HQ), S. Horowitz (NASA/GSFC), and P. Tyler (NASA/GSFC).

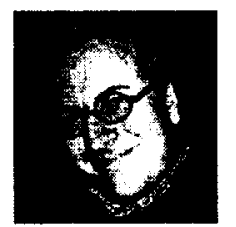

Nicholas White is the Chief of the Laboratory for High Energy Astrophysics at NASA's Goddard Space Flight Center. $\mathrm{He}$ is the senior scientist for the SEU theme. He has over 100 refereed publications in the field of $x$-ray astronomy. He is also Director of the High Energy Astrophysics Science Archive Research Center (HEASARC) 
Bruce Margon is a professor of astronomy at the University of Washington, Seattle. He is chair of the SEU subcommittee that advises NASA on the strategic planning of its missions. 